\title{
A Study on Oriental Aesthetics in Pictorialism*
}

\author{
Bin Guan \\ Hubei University of Education \\ Wuhan, China
}

\begin{abstract}
Pictorialism as a photography branch which achieves aesthetic conception through documentary mode, its essence has a link with Chinese calligraphy and painting, and they all are Oriental arts with typical Oriental aesthetic. And artistic conception is the highest level of oriental aesthetics, also is a perfect form of expression for pictorialism to pursue. Although pictorialism relies on documentary way to innovate, but it is carrying Chinese culture and national connotation, the aesthetic category it contained reflects the aesthetic form of the work. This article uses "shape and spirit", "dynamic and static", "reality and fantasy, "light and shadow" to render the artistic conception in pictorialism, and to explain the connotation and formal beauty of oriental culture as well as its symbolic meaning.
\end{abstract}

\section{Keywords—oriental aesthetics; photography; pictorialism}

\section{INTRODUCTION}

Zong Baihua once said: "The fusion of nature and heart has become a crystal of life, the kite fly in the air and fish jump in the water, exquisitely carved, this is artistic conception, the center of all the arts' center. Artistic conception is the unity of nature and heart. "Since it is pictorialism so naturally it can not get away with "artistic conception", not to mention the pictorialism and Oriental aesthetic category have the same strain. The aesthetic style of pictorialism and the oriental aesthetic concept it has make people's vision more broad and enhance their own spirituality at the same time. Use the harmony between man and nature to express a deeper level of emotion and make sublimation to beauty. The artistic conception is the highest realm of oriental aesthetics, and it is a perfect form of expression that pictorialism pursues. It makes the idea of the photographer and the spirituality of the object combined together, so that all things in the photo expressed their spirituality and beauty. The performance of pictorialism is like a picture with rich and far-reaching meanings, it has a kind of feeling that there is fragrance come from dark. The expression mode of pictorialism reflects the height of art and the general realm of thought, which brought a broad imagination space to the viewer. So it has a realm of art which beyond the reality beyond the specific appearance in front of you and perfectly combined feelings and scenes together.

*Fund Project: (This article is one of the results of Humanities and Social Sciences Research Youth Fund Project of Hubei Provincial Department of Education in 2016 "Application research of 3D photography in digital museum construction ",Project approval number:" 16Q272 ").

\section{THE APPLICATION RESEARCH OF ORIENTAL AESTHETIC IDEA IN PICTORIALISM}

Oriental aesthetic idea has very profound impact on pictorialism, which is reflected in almost all the pictorialism works, regardless of the main body of the picture, or the different scenes that been replaced, they reflect more or less the artistic conception of Oriental aesthetics in pictorialism. And in this context, this article mainly refers to a few traditional characteristics of Chinese traditional aesthetics, so that the viewers could think about pictorialism works from the Oriental aesthetic point of view, and experience the profound Chinese traditional aesthetics. Whether it is ancient or modern, every artwork can be explained its beauty from the aesthetic point of view, this distinctive aesthetic feature is also an important performance of pictorialism.

\section{A. Discuss "Form" with "Spirit"}

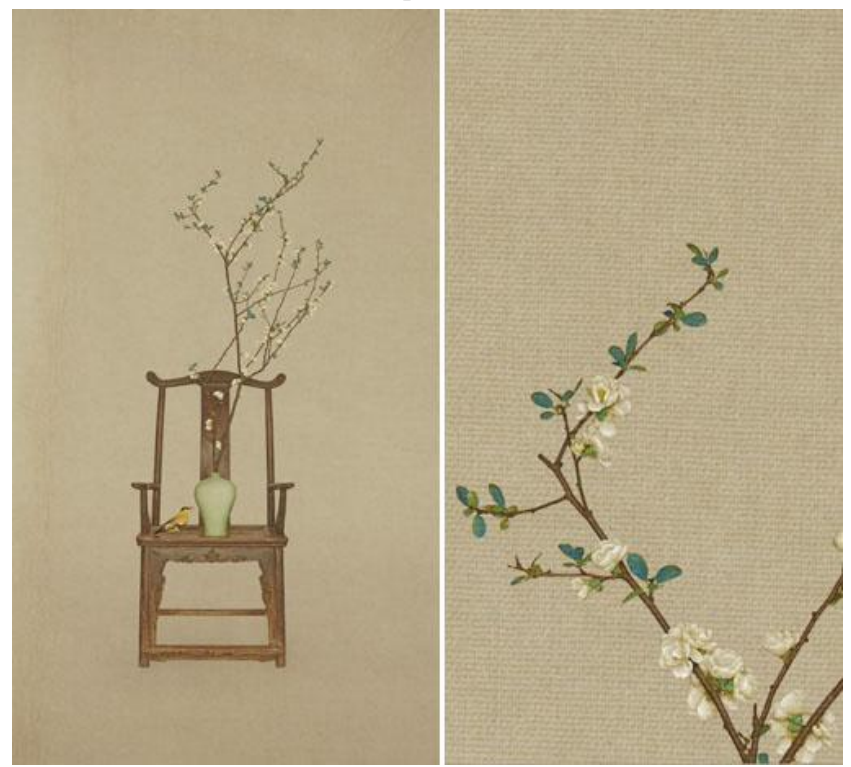

Fig. 1. White Crabapple in jade vase. (The picture is from www.sunjunphoto.com) 


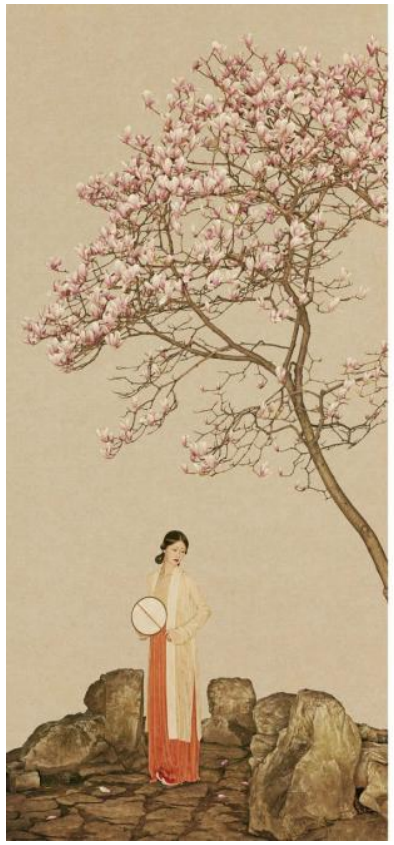

Fig. 2. Late spring of the four verses in Song Dynasty. (The picture is from www.sunjunphoto.com)

"Form and spirit" is a basic category of Chinese aesthetics. As the name implies, in the range of aesthetics, form is the shape of foreign objects, spirit is a kind of charm, the two complement with each other and indispensable. In pictorialism when "spirit control form"" is used to express an inward, subtle beauty in nature. As shown in "Fig.1", we found in Mr. Sun Jun's photo of "White Crabapple in jade vase" that although there are only one flower, one bird and one chair in the photo, but it is simple yet with flavor, shape of flower branch, bird's demeanor are placed in the eyes of viewer, as if there are stories with thousands of turns and twists. So that although the screen is a still space, but viewer's feelings are floating fragrant, implied with vitality. In such an antique spiritual world, it seems there are infinite flavor of "Danji shizhi huagengyan, chouduo yande yuwuhen. Yuchang baidi yiqingjie, buyu tingting riyouhun." (when you get used to plain color, you will know how bright color does the prosperous. There is no way your face did not have the time trace if you have so many sorrows. The white crabapple would like to return to nature as its clean body, her stand beautifully and silently, day after day). Your face will have in the air. The emotional thoughts that are intended to be expressed in the picture are embodied in the unique rich connotations of pictorialism and the implication of overflowing words.

So, the performance of form and spirit can also be in the combination between material and people, as shown "Fig. 2" Mr. Sun Jun's "Late spring of the four verses in Song Dynasty", this figure brought a gentle melancholy sense into the eyes of viewer. In the picture, Magnolia comes from the right side, tilted upward like the woman under the tree whose heart is flying toward the sky. The woman is holding a cattail leaf fan, lower her head and brows seem like she is talking about the meaning of "Yijiu bukanwen, jiangnan huangguan geqiuyun. Duoqing bugai niannianse, qiangu fangxin chizengjun" (Time passed by, soon the new poem is now good to hear, there is a house somewhere in Jiangnan may has tree shadow wave, but the flower is still blossom every year, this long-lasting noble flower is exactly the thing to send to a gentlemen as gift )This kind of connection and link between scenery and object, people and material make simple picture is meaningful. Still is one tree one person one stone one fan, but also with a rich spiritual world. The idea of form and spirit in Oriental aesthetic embodied in pictorialism, probably has a kind of state like Zhu Da said "Jingchi fenglu buqiuzhi, ziyou hengxie baibianzi. Liude shenshan youyuzai, kenjiao yanmo tailinli "(The flower has thousands of gestures, its elegant flavor will make ink too heavy)

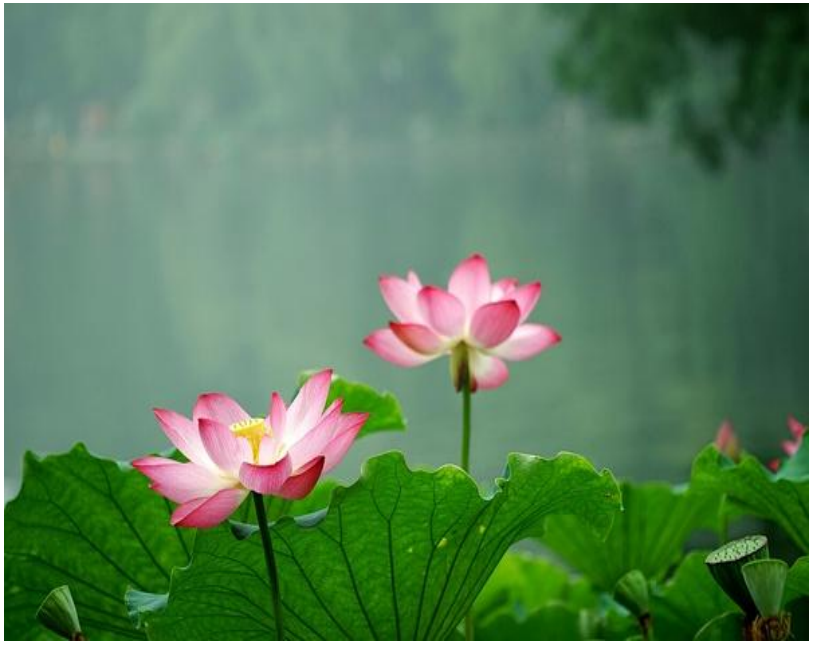

Fig. 3. Lotus. (The picture is from zhujian, www.douban.com)

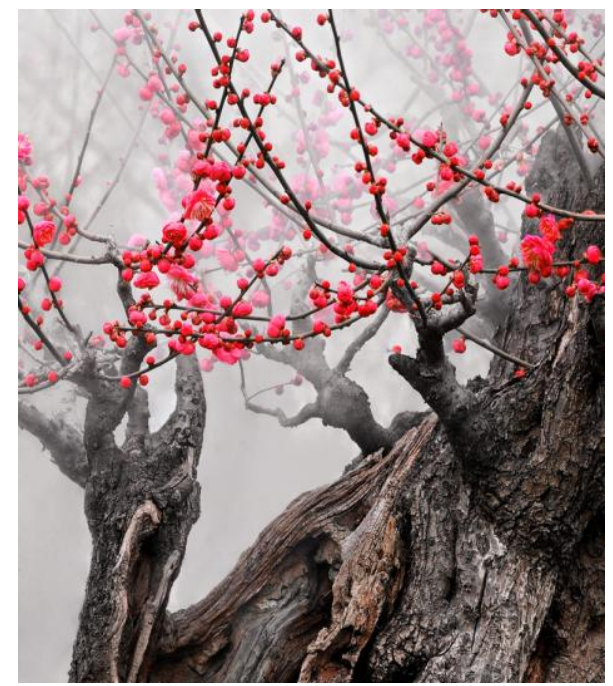

Fig. 4. Plum. (The picture is from www.baidu.com)

Pictorialism is also common in life, many works can express their own emotions, so that their minds have a home and the works could reach a realm with both form and spirit. For example in "Fig. 3" this work, the screen atomized lotus background, the two lotus in the middle one behind the other 
highlight the delicate and charming of lotus. In the atomized background, the charming lotus and the refreshing lotus leaves make them own their own small world and write their own beautiful poetry: "Kauihua zhuoshuizhong, baoxing yihejie! Zhulan yuemingzhong, qingxiang weisheifa" (Lotus blossom in dirty water, but it keeps its noble honor! When in the red fencing under the bright moon, whom does it release the fragrance for?). Lotus is the flower with spiritual aura, since ancient times it has been the preference of the literati and refined scholars, is because lotus has a mysterious and desirable character. The theory of form and spirit is more like this.

And "Fig. 4", the subject of this work is no doubt plum, it is arranged in the white fake looming, in order to bring out the bright and red of plum blossom, sturdy trunk is used to stabilize the picture, so that the picture won't appear to be light because of white fog. The picture is also clean and concise, with only three objects, it highlights the theme and improves the attractiveness of the work. The white fog which occupies one-third of the picture added mysterious atmosphere for the work, and pretty plum blossom brought vitality to the work. This picture makes people hold their breath and focus on it, it makes them feel like in a winter morning, and he smells the fragrance of plum blossom and travels in his imagination world. The form and spirit problem of Pictorialism is not stiff copy the nature, but to obtain the "spirit" and not left the "form" and retain the essence between nature and art, as far-reaching and deep as "Yaozhi bushixue, weiyou an'xianglai" (You can tell it is not snow from a distance is because you can smell the fragrance of it).

\section{B. Express "Dynamic" with "Static"}

Express "dynamic" with "static" shows the rhythm of life outside static. Its pursuit is an overflow of a clear stream of like in our quiet state. Express a lively spirit of life in the quiet, static and dynamic combined together making no difference between "you" and "me". The picture of pictorialism is a space to show life, everything in the world has its own life tension.

"Fig. 5" is Sun Jun's photo "Dayinxisheng". The photographer poet grasped the pulse of life, in the bamboo forest, bamboo grow quietly with its own posture, although static, but vivid. Three pieces of big stone scattered together, although they are heavy, but also it and the light wild flower can the help each other when both are in humble circumstances, along with the flow of time to release the vitality of life. On the stones there are two persons, one is dancing and other is plying Sheng, it seems they are singing for the creatures of this land and dancing for the spirit of life for everything. The picture is still, can actually it uses be static to express dynamic: describe the tine flow, life, music, telling the sound which remained lingering around the beams for three days, singing a feast of life rhythm.
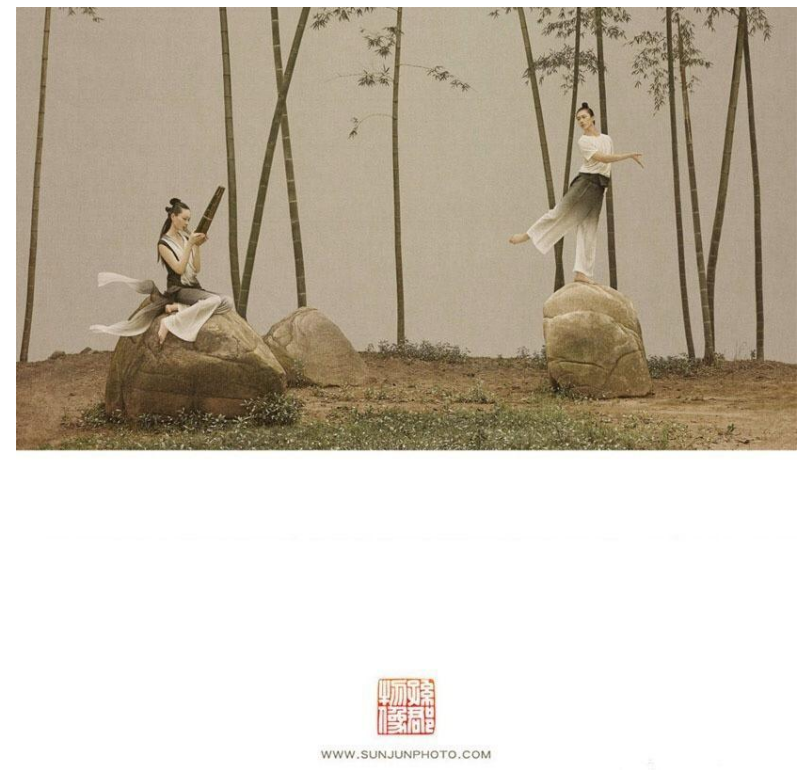

Fig. 5. The greatest sound is silence. (The picture is from www.sunjunphoto.com)

"Fig. 6" is also took by Sun Jun, called "The rhythm of life", the picture is ethereal silence, the big tress fallen on the ground symbolizes the beginning of new life, rather than the end. The branches on the right side dancing with the flute, the dancing is the tension of life, it supposedly is what Shi Huiyuan said: "Xisheng zouqunlai, xiangchu shanliudi. Youke dumingyou, jingran wangsuoshi." (The silence here is just like all sounds come out together, this sound is like water drops falling from mountain.I am here touring while thinking about life, and almost forget where I am now).In this nature with hidden rhythm , forget itself, just enjoy the still and restless life. This is not a static space, it shows the concern for dynamic. Romantic feelings fly to heaven because life is dancing. Feel clear song and the turbulence of new life is the meaning of this work of "The rhythm of life", and express "dynamic" with "static" is the meaning of Oriental aesthetic in pictorialism.

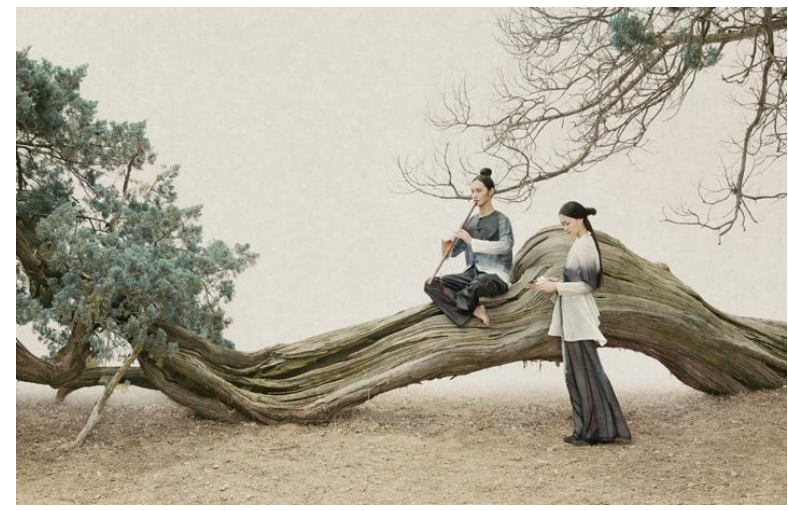

Fig. 6. The rhythm of life. (The picture is from www.sunjunphoto.com)

From the perspective of the Oriental aesthetic concept, express dynamic with static mainly use the concept of static and dynamic coherent in order to show the meaning which 
the picture want to express. It is a harmonious state. The picture should not have too many things to prevent the screen is too full to be crowded, it is contrary to the basic concept of beauty. Second, when select the subject, you should know how to extract works if you want to show beauty. Oriental culture is broad and profound, natural scenery is rich and colorful, so the picture should be clean and simple but with content.

There are mountain, forest and birds in the picture "Fig. 7', but trees only take one-third of the picture, left two-thirds of the space to the birds. Such framing will not be rigid, and the feelings it brought to people will not be uncomfortable. In the artistic conception of static and dynamic, there is a feeling of tired birds fly back, sunset flow on the sky, the dawn is the best view of the world. The picture could be static and dynamic, harmonious and quiet. In pictorialism, this photo both has a sense of emotion and a broad-minded feeling, as well as exciting moments.

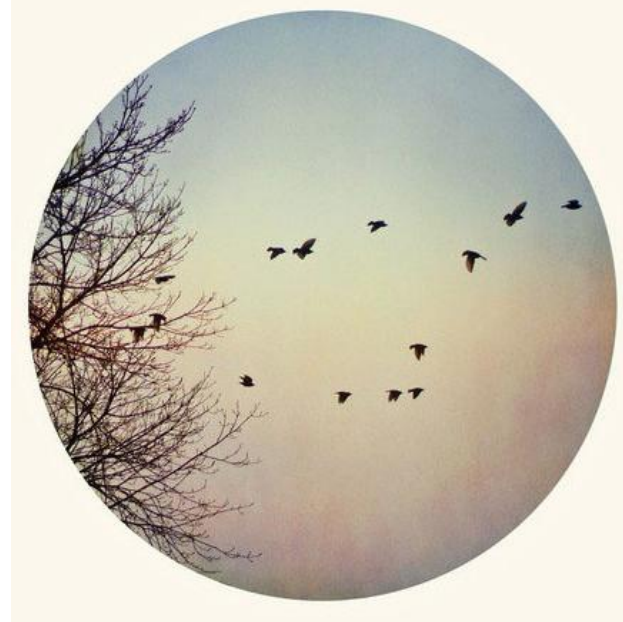

Fig. 7. Mountain, forest and birds. (The picture is from www.baidu.com)

"Fig. 8" is also shows an intuitive picture of static and dynamic. The sea is smart and ever changing. Although the picture is lack of the momentum of usual Sanwanlihe dongruhai" (thirty thousand miles river flow into the sea) and "Junbujian huanghezhishui tianshanglai, benliudaohai bufuhui"(You don't see that the Yellow River comes from sky and flow into sea with no returns). But it has extra attitude of "Haishui wufengshi, botao anyouyou"(when there is no wind at sea, the wave is peaceful), and the disappointed distance watching of "Baichuan dongdaohai, heshi fuxigui" (All the rivers flow into the sea, when will them return to the west).The sand is quiet and settling. Under the ocean's rising, falling tide, sand uses its quiet, left a beautiful image of seawater surging to the viewer. Just under this combination between static and dynamic, so that the picture is touching yet elegant.

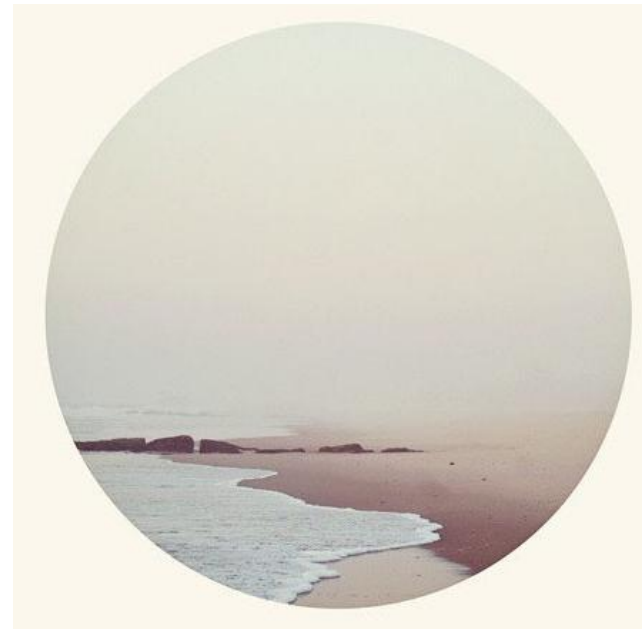

Fig. 8. Thirty thousand miles river flow into the sea. (The picture is from www.baidu.com)

\section{Contrast "Fantasy" with "Realty"}

Du Mu had a poem: "Yanlong hanshui yuelongsha, yebo qinhuai jinjiujia. Shangnv buzhi wangguohen, gejiang youchang houtinghua"."(Blurred moon light and light smoke covered the cold water and white sand, the boat anchored near the Qinhuai river at night. The singing women did not know what is the hate of a conquered country, still singing the song "Houtinghua" acrossed the river) The whole poem has a feeling like a trace of smoke, seems like or not like,keeping apart but not afar, with a lonely charming color. Then look at the boat in water, interweave reality with fantasy, with a far-reach meaning. For the concept of reality and fantasy in Oriental aesthetic concept, it is like this poem, reality mixed with fantasy, reality and fantasy lived together. In pictorialism you could lead to a new realm if you can achieve the contrast reality with fantasy.

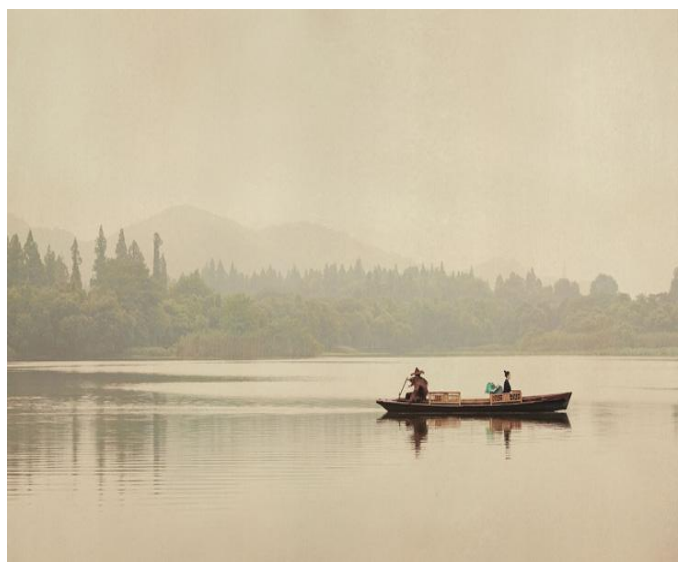

Fig. 9. Small boat. (The picture is from www.baidu.com)

Take "Fig. 9" Sun Jun's photo "Small boat" as an example. The river was calm, only water ripples left by the boat cleanse the bottom of people's hearts in the boat. Mountains piled one on the other was covered with a layer of fog when it was in sight, quite the taste of fireworks mixed with mountains, among which the sense of loss and 
confusion are naturally integrated into this mountain scenery. The reflection of the calm water was so misty and illusory, the reflection of the boat and people is just like moon in water, flower in mirror. As the poem said, "Leizhu quanbeixia, choulian chudisheng"(wine and music both are the representative of sorrow, good friend will leave soon so blow the fluent for him to express the missing emotion), a thousand words I do not know where to start, only express my feelings with a fluent song. It is a regret which will be broken after a careless touch. These subtle, misty, soft scenes contrast the only real scene in the picture, boat. The boat is like the last sentence of the whole poem,which attracted all the focus of people on it, because it is the result of the former bedding, is the finishing touch. In this fantasy and reality, the implicit beauty is infinite, so it is an other fine work of pictorialism using aesthetic concept.

Distant layers of mist set off a paradise atmospheruree for the entire picture "Fig. 10". It makes people want to escape from the reality and walk into this artistic fairyland with full of pleasure. The introduction of smoke clouds also makes the peach blossom forest extraordinarily "Liaoluo guxinggong, gonghua jimohong"(in the empty and cold ancient palace, only flower blossom lonely), in order to contrast the focused place could enter people's heart and make the whole work more interesting. The reason why the concept of reality and fantasy used in pictorialism makes so interesting, is because it fit with the implicit beauty and silent beauty in Oriental aesthetics. Only such implicit implied, looming existence could make the picture more fascinating.
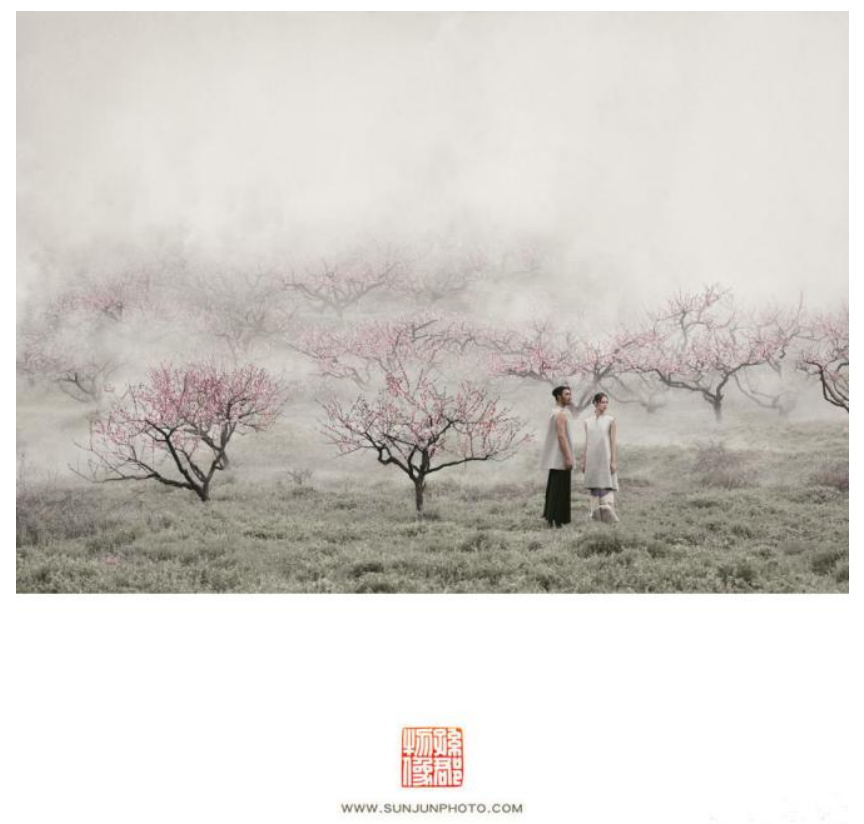

Fig. 10. Distant layers of mist. (The picture is from www.baidu.com)

If there is no real scene on the right side "Fig. 11", even though how poetic and artistic the whole picture is, it will make the dawn and watery towns in Southern China together in the sleeping spell and never woke up. The whole work seemed to be filled with white blue light, like a cold and charming picture scroll. People tend to love implicit, mild blurred things. But too blurred, the whole picture is in the fog without real object, will also make people disappointed and could not bear to see it. This is the importance of the aesthetic of reality and fantasy. Pictorialism actually is a combination of realistic and imagination. Like "Fig. 12", the lake flashed the shadow of the boat, pure the landscape into the bottom of the lake like ink, the people on the boat and this picture, among which the theory of reality and fantasy has a common place in here.

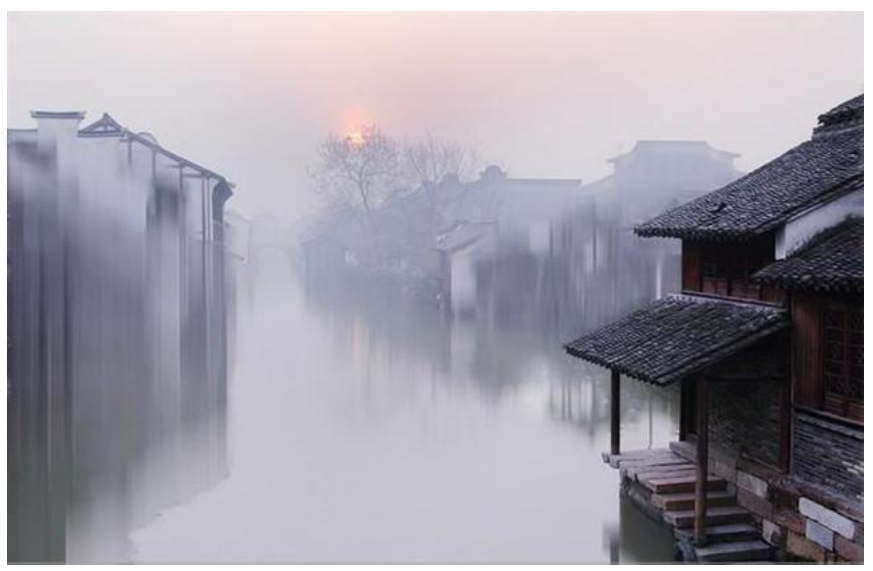

Fig. 11. Watery towns in Southern China. (The picture is from www.baidu.com)

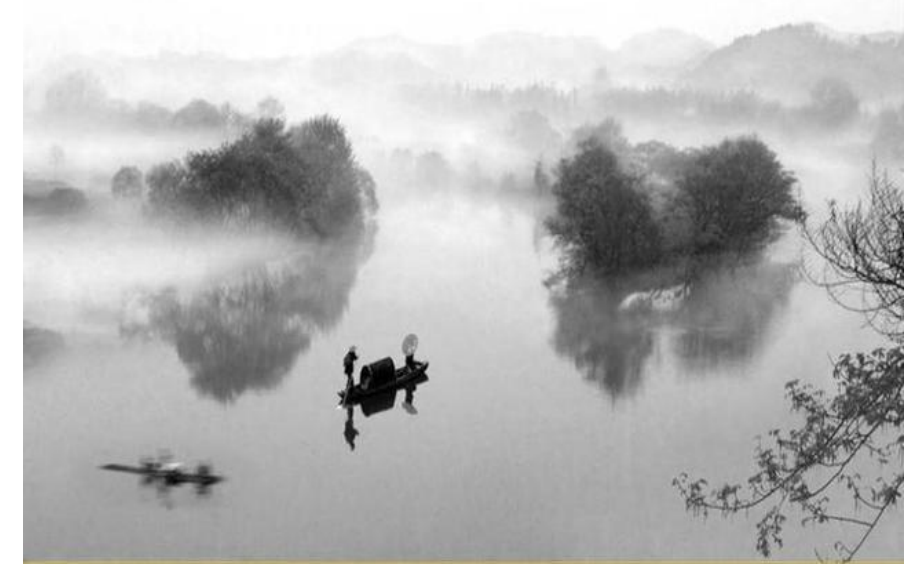

Fig. 12. The lake flashed the shadow of the boat. (The picture is from www.baidu.com)

The reality and fantasy concept of Oriental aesthetics in pictorialism plays the role of atmosphere creation, spreads slowly between the realty and fantasy, alternate tension with relaxation, bring people to the artistic conception of space. It uses indirect rendering with the fantasy part and then set off the focused place in order to make the perfect final touch. In this ambiguous space-time conversion, the real place is the center of the picture, it has a role to highlight the whole piece of work just brilliant writing. This kind of express Oriental aesthetics concept with the artistic conception of space through the reality and fantasy uses in actual situation to 
bring the mood to express the East Senmei aesthetic concept used in pictorialism, really full of flavor.

Mr. Zhu Guangqian once said: "as art can not be apart from perception and imagination, it can not be apart from association." Light and shadow is an art of time and space, it is because of the application of light and shadow in pictorialism, so that it makes pictorialism has an infinite charm in time and space. Like the "blank-leaving" in Chinese ink painting, blank-leaving is a typical oriental aesthetic feature, it left a blank in the picture based on the creation object in order to allow the viewers to imagine, associate to the interests beyond the picture. Light and shadow make the viewer to feel three-dimensional in a plane object, feel the far distant, feel the emotions and thoughts after break away from the real object.

"Fig. 13" is a photo that uses light and shadow to express the beauty of space. The whole picture is a closed dream space, mottled light of trees projected on monks and white wall, as if enveloped in a quiet space, but also like a golden fleeting time with full of poetic and pictorial splendor. It is fallen sun above a long river, it is solo smoke in the desert, it is ten year's meditation in morning bell and evening drum, it is the peace and enrichment under green light and ancient Buddha. This is light and shadow, it resonate with photography under the most suitable and most appropriate circumstances, the blank it left has left enough imagination space for the view to enjoy the artistic conception of ten years dream which brought by the virtual, flowing space.

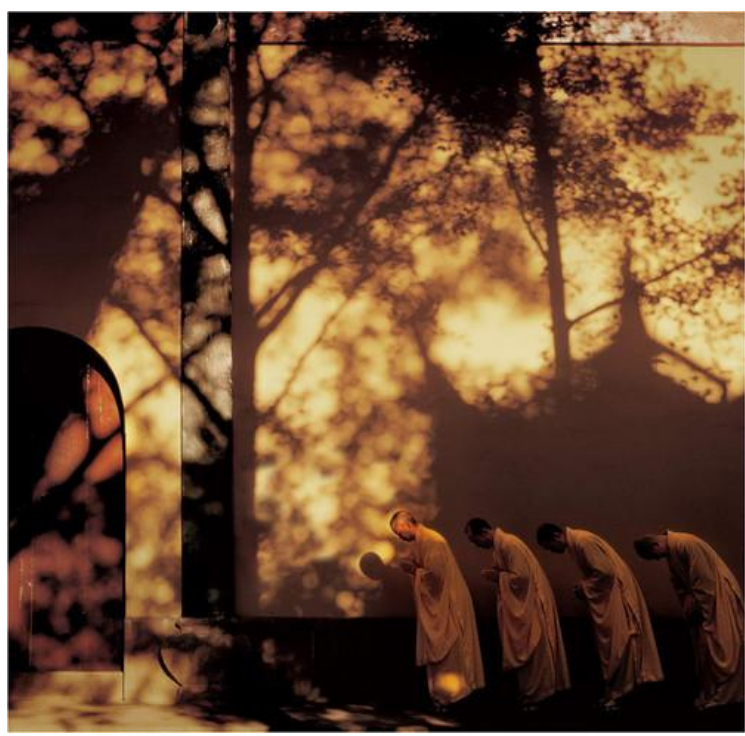

Fig. 13. Buddha's footstep-Disillusionment. (The picture is from www.baidu.com, shot by Zhang Wang)

"Fig. 14" the picture uses the temple's wooden door, monk's profile, the censer and smoke outside the door, mountain fog, the empty silence inside the temple to develop layers of space bedding under light and shadow, which have broken the screen restrictions. Followed by the view's eyes, move to the outside of the picture along with the objects, to associate with an infinite space. These artistically described the strong poetic feelings, and also express the association beyond the current world which brought by the picture. The fast passage of time is so meaningful in the picture, which lead to deep thought and sign. However, this "silent and farreaching" picture style which brought by light and shadow has common sense with the aesthetic image which brought by the "blank-leaving" of Chinese ink painting.

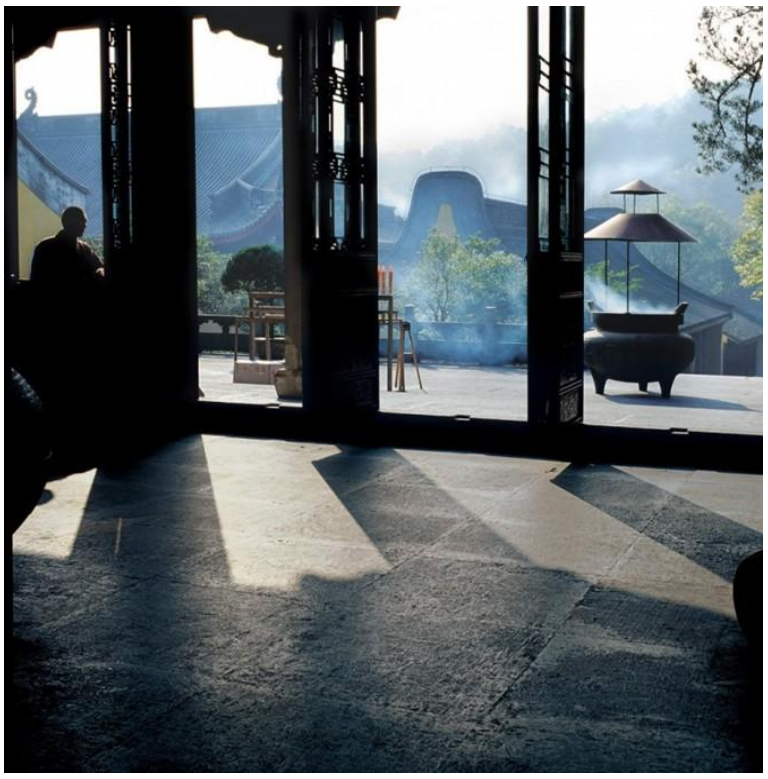

Fig. 14. Buddha's footstep-Time. (The picture is from www.baidu.com, shot by Zhang Wang )

The spatial awareness brought by light and shadow embodies unique oriental aesthetic characteristics: they are charm also there are lonely, but moreover they are profound and open-minded. These are not only the poet's poetry but also the artistic conception of pictorialism.

From the Oriental aesthetic point of view, light and shadow played a role which handle complicated matters with ease in photography. Using it in pictorialism not only is in line with the characteristics of oriental aesthetics, but also adds a new color to pictorialism, so that it has an aesthetic spirit without restraint and full of the power of silence.

\section{CONCLUSION}

Through the understanding of pictorialism, the cognition of Oriental aesthetic ideas and its application in pictorialism, to know this art of photography that has its own unique artistic conception. But the pictorialism should keep improving during its growing process; Among which, reasonable use of oriental aesthetic concept is the basic premise, enhance the photographer's technical ability is the key factor, bring us beautiful works and at the same time teach everyone the correct aesthetic way is the final purpose. Only when reasonably integrating the aesthetic characteristics to express the photographer's subjective intentions, emotions and feelings on the basis of correct understanding of pictorialism, we could create a pictorialism work with aesthetic prospect and rich aesthetic image. 


\section{REFERENCES}

[1] Chen Yunlu: "Listen to Zhu Guangqian about aesthetics", Anhui People's Publishing Company. March 2012.

[2] Du Fu, Note by Qiu Zhaoyi, paint by Lu Yanshao: "Du Fu poetry illustrated book", Zhejiang People's Fine Arts Publishing Company, August 2014.

[3] Shen Deqian (Qing), proofed by Sun Tonghai : "The source of Ancient poetry", Liaoning Education Press. March 1997.

[4] Tan Yonggang: "Chinese painting ' form.spirit' - static and dynamic theory", Art gallery. January 2012.

[5] Wang Guoying: "Chinese landscape poetry research", Baihua Literature and Art Publishing Company, 2011 edition.

[6] Zhu Guangqian: "Silent beauty", Peking University Press, January 2005.

[7] Zhu Liangzhi: "Qu Yuan Feng He - Ten talks on Chinese art ", Anhui Education Press, January 2010. 